\title{
Autonomous Navigation System of Hexapod Robot based on Fuzzy Neural Network
}

\author{
Li Shuang-hong ${ }^{a}$, Kong Ling-wen ${ }^{\mathrm{b}}$ and Du Qiao-ling* ${ }^{* \mathrm{c}}$ \\ School of electronic Science and Engineering, Jilin University, Changchun 130012, China

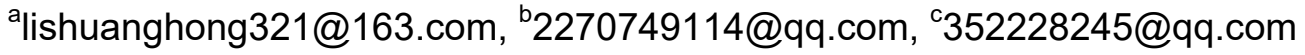

Keywords: Hexapod robot; Fuzzy neural network; Closed-loop ; Autonomous navigation.

\begin{abstract}
A closed-loop autonomous navigation system based on fuzzy neural network is proposed to deal with the autonomous navigation issue of hexapod robot in unknown environment. The closed-loop system is designed in order to optimize the output performance. The navigation algorithm is designed to combine fuzzy control with neural network. Fuzzy control is used to realize the ability of logical reasoning and neural network is conducive to learning and training ability. The ambient sensors are a GPS sensor, an electronic compass sensor and an ultrasonic sensor with sector scanning state. These sensors can complete the detection of the surrounding obstacles, the target course angle and the current course angle. The performance of the robot's autonomous navigation system is compared with the open-loop and closed-loop system based on fuzzy neural network in the simulation experiment, which demonstrates that walking time based on closed-loop system significantly declines compared to the open-loop system, meanwhile, the traveling speed also improves. In the way to the destination, the robot can safely and quickly bypass the obstacles without any redundant paths.
\end{abstract}

\section{Introduction}

Robot autonomous navigation is one of the core technologies in mobile robot research. It plays an important role in many fields such as industry, agriculture, service, medicine. At present, the study of robot autonomous navigation tends to develop intelligently, and some navigation algorithms have been proposed, for example neural network[1,2], fuzzy control[3,4] and the combination of fuzzy control and neural network[5]. The neural network has a strong ability to learn and train, but lacks processing and description capabilities of fuzzy information. The fuzzy control has a logical reasoning ability, but the weak learning and adaptive ability limits its application in unstructured environments. The combination of fuzzy control and neural network can make the system has both the ability of learning, training and fuzzy reasoning ability. Nevertheless, the development of algorithms for robot autonomous navigation are still based on an open-loop control method, which is weak in path selection and speed control of robot relative to the closed-loop control method[6].

In this paper, A closed-loop system is constructed by adopting BP neural network as the basic framework. The fuzzy logic layer and the feedback function layer are added to the closed-loop control system. The feedback signal of the closed loop control system is the output speed and the steering angle. Navigation algorithm based on fuzzy neural network modify the input information and adjust the weights of the neural network through the multiple training and learning of the samples to ensure the convergence speed and stability of the whole system. The system realizes the safe and fast autonomous navigation function of the robot.

\section{Kinematics Model Analysis}

Environmental information acquisition and optimization. The mechanical structure of the hexapod robot is shown in Fig.1. The robot's perception of the surrounding environment is realized by sensors, therefore the types and distribution of sensors are very important for the whole system. This paper selects ultrasonic sensor, GPS sensor and electronic compass sensor as the perceptrons. In order to solve the problem that ultrasonic sensor can not accurately obtain the boundary 
information of obstacles because of the presence of ultrasonic beam angle $\left(30^{\circ}\right)$, we adopt a ultrasonic sensor to do the sector scan as shown in Fig.2. The ultrasonic sensor is designed to turn 5 times[7,8], each time at a fixed rotation of $30^{\circ}$, in order to detect obstacle distance information in 5 directions. This detection scheme allows the robot to detect obstacles in $120^{\circ}$ in the direction of travel, basically consistent with the visibility of the human eye. The combination of GPS sensor and the electronic compass sensor is designed to measure the location information of the robot in this paper[9]. The GPS sensor is used to detect the target course information of the robot and the electronic compass sensor is used to detect the current course information of the robot.

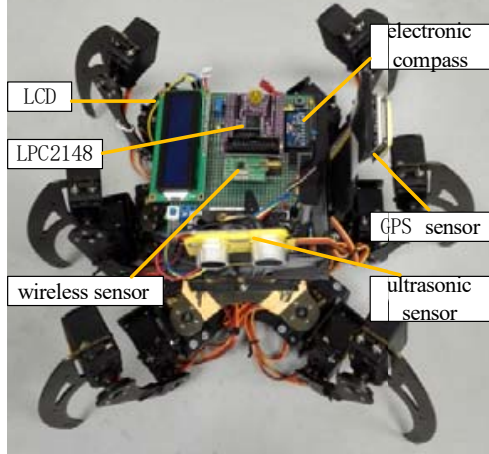

Fig. 1 Mechanical structure of hexapod robot

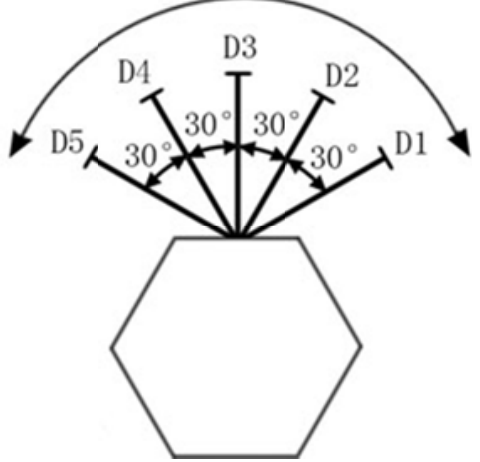

Fig. 2 Sector scan of ultrasonic sensor

Establish the robot kinematics model. A kinematic model of robot is established in the two-dimensional reference coordinate system, as shown in Fig.3. The coordinate of the starting point of the robot is $\left(\mathrm{X}_{0}, \mathrm{Y}_{0}\right)$, and the coordinate of the target point is $\left(\mathrm{X}_{\mathrm{G}}, \mathrm{Y}_{\mathrm{G}}\right)$. The coordinates of the robot is $\left(\mathrm{X}_{\mathrm{t}}, \mathrm{Y}_{\mathrm{t}}\right)$ and the velocity is ' $V_{t}$ ' at time ' $\mathrm{t}$ ', in which ' $\mathrm{V}_{\mathrm{t}}$ ' and ' $\theta_{\mathrm{Vt}}$ ' are absolute speed and course angle. So the coordinates of the robot is $\left(\mathrm{X}_{t+1}, Y_{t+1}\right)$ and the velocity is ' $V_{t+1}$ ' at time ' $t+1$ ' , in which ' $\mathrm{V}_{\mathrm{t}+1}$ ' and ' $\theta_{\mathrm{Vt}+1}$ ' are absolute speed and course angle. The target distance and target course angle of the robot can be expressed by ' $\mathrm{E}_{\mathrm{t}}$ ' and ' $\theta_{\mathrm{Et}}$ ' at time ' $\mathrm{t}$ '.

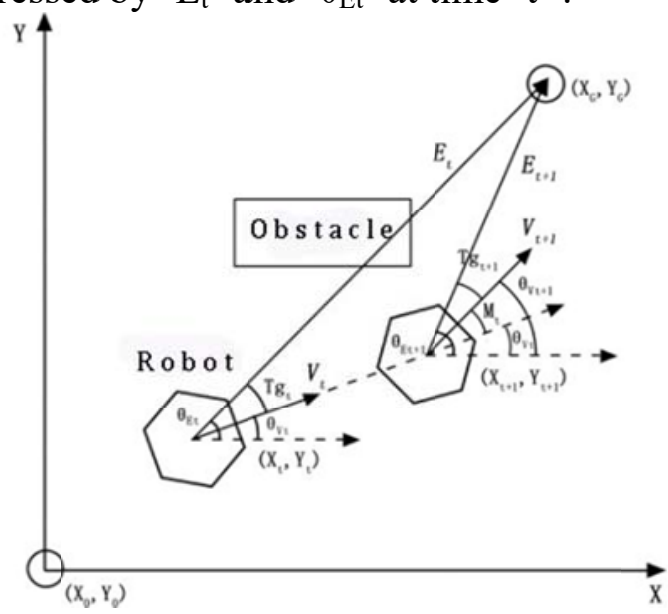

Fig.3 Kinematics model of robot

$$
\begin{aligned}
& E_{t}= \sqrt{\left(X_{G}-X_{t}\right)^{2}+\left(Y_{G}-Y_{t}\right)^{2}} \\
& \theta_{E t}= \begin{cases}\arctan \frac{Y_{G}-Y_{t}}{X_{G}-X_{t}} & \left(\left(X_{G}-X_{t}\right)>0\right) \\
\arctan \frac{Y_{G}-Y_{t}}{X_{G}-X_{t}}+180^{\circ} & \left(\left(Y_{G}-Y_{t}\right)>=0,\right. \\
\arctan \frac{Y_{G}-Y_{t}}{X_{G}-X_{t}}-180^{\circ} & \left(\left(Y_{G}-Y_{t}\right)<0\right) \\
90^{\circ} & \left(\left(Y_{G}-Y_{t}\right)>0,\left(X_{G}-X_{t}\right)=0\right) \\
-90^{\circ} & \left(\left(Y_{G}-Y_{t}\right)<0,\left(X_{G}-X_{t}\right)=0\right) \\
0^{\circ} & \left(\left(Y_{G}-Y_{t}\right)=0,\left(X_{G}-X_{t}\right)=0\right)\end{cases}
\end{aligned}
$$

$\theta_{E t} \in\left(-180^{\circ}, 180^{\circ}\right]$
(1)

$$
\begin{aligned}
& X=(N+H) \cos B \cos L \\
& Y=(N+H) \cos B \sin L \\
& Z=\left[N\left(1-e^{2}\right)+H\right] \sin B \\
& N=\frac{a}{\sqrt{1-e^{2} \sin ^{2} B}} \\
& e^{2}=\frac{a^{2}-b^{2}}{a^{2}} \\
& T g_{t}=\theta_{E t}-\theta_{V t} \quad \theta_{V t} \in\left(-180^{\circ}, 180^{\circ}\right] \\
& \theta_{V t+1}=\theta_{V t}+M_{t} \\
& T g_{t+1}=\theta_{E t+1}-\theta_{V t+1}=\theta_{E t+1}-\theta_{V t}-M_{t}
\end{aligned}
$$


In Eq.(1), ' $\mathrm{X}_{\mathrm{G}}$ ', ' $\mathrm{X}_{\mathrm{t}}$, ' $\mathrm{Y}_{\mathrm{G}}$ ', ' $\mathrm{Y}_{\mathrm{t}}$ ' are the location information of the GPS sensor, which is obtained by transferring the latitude and longitude information from the geodetic coordinate system into rectangular coordinate system in Eq.(3). Since the robot motion is restricted to the surface plane, we only use (Y, X) coordinates as the robot's two-dimensional coordinates. In Eq.(3), ' $\mathrm{L}$ ', ' $\mathrm{B}$ ' and ' $\mathrm{H}$ ' denote the longitude, dimension and height of the measured point, ' $\mathrm{X}$ ', ' $\mathrm{Y}$ ' and ' $\mathrm{Z}$ ' denote the position coordinates of the measured point in the space rectangular coordinate system. ' $\mathrm{Tg}_{\mathrm{t}}$ ' represents the angle between the target course angle and the current course angle at the current time ' $\mathrm{t}$ ' in Eq.(6), in which the current course angle ' $\theta_{\mathrm{Vt}}$ ' of the robot is measured by the electronic compass sensor . ' $\mathrm{M}_{\mathrm{t}}$ ' represents the steering angle of the robot at the current time ' $\mathrm{t}$ ' in Eq. $(7,8)$, which is one of the output information of the navigation system, then the course angle and the target angle of the robot at time ' $\mathrm{t}+1$ ' ' are expressed by ' $\theta_{\mathrm{Vt}+1}$ ' and ' $\mathrm{Tg}_{\mathrm{t}+1}$ '. It can be seen that the output steering angle ' $\mathrm{M}_{\mathrm{t}}$ ' at current time ' $\mathrm{t}$ ' is fed back to the input terminal, and the input target angle information ' $\mathrm{Tg}_{\mathrm{t}+1}$ ' at time ' $\mathrm{t}+1$ ' is corrected and adjusted.

The definition of kinematic model. Definition 1: The input state space vector ' $x$ ' of the hexapod robot. Definition 2: The output state space vector ' $s$ ' of the hexapod robot.

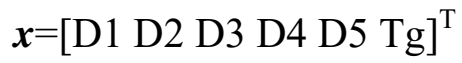

$$
\begin{aligned}
& \boldsymbol{s}=[\mathrm{V} \mathrm{M}]^{\mathrm{T}}
\end{aligned}
$$

Where 'D1', 'D2', 'D3', 'D4' and 'D5' are distance information of obstacles captured at five angles $(-60,-30,0,30,60)$ of the ultrasonic sensor, ' $\mathrm{Tg}$ ' is the angle information of robot between the target course angle and the current course angle. ' $\mathrm{V}$ ' represents the output speed of the hexapod robot, and ' $\mathrm{M}$ ' represents the steering angle of the hexapod robot in the system.

The safety performance analysis of the robot. The walking safety of robot is very important in the autonomous navigation system. The problem of "too close" will occur when the robot walks close to the edge of the obstacles or turns[10], so the robot needs to keep a safety distance with the obstacles to turn around. In this paper, the safety distance is defined as 'SD', and the relationship between the safety distance ' $\mathrm{SD}$ ' and the current velocity ' $\mathrm{V}_{\mathrm{t}}$ ' is shown as follows:

$$
S D>=\left(V_{t}+V_{\max }\right) * \tau+R
$$

Where ' $\mathrm{V}_{\max }$ ' is the maximum traveling speed of the robot and ' $\mathrm{V}_{\mathrm{t}}$ ' is the traveling speed at current time ' $t$ '. The value of ' $\tau$ ' is ' $\lambda$ ' seconds, in which ' $\lambda$ ' $(>=1)$ is the safety threshold and the value is ' 1 ' according to the actual demand. The maximum radius value of the robot body is ' $R$ ' which is the distance between the outermost point and the center of the robot. In this paper, the safety distance is changed in real time with the change of the robot moving speed ' $\mathrm{V}_{\mathrm{t}}$ ', which avoids the problem of path redundancy caused by a single defined safety distance, and improves the real-time and flexibility of the system.

\section{The Design of Hexapod Robot Closed-loop System}

In this paper, the BP neural network and fuzzy control are combined to design a closed-loop fuzzy neural network system as shown in Fig.4.

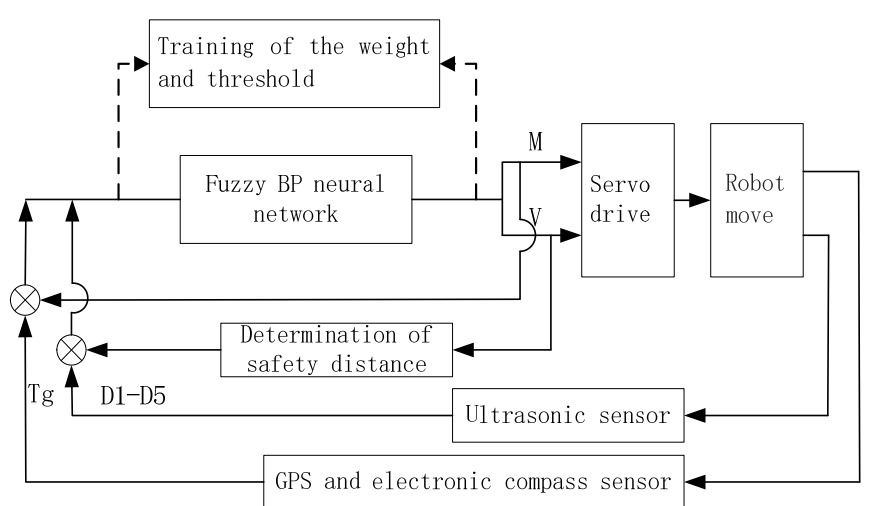

Fig.4 Closed-loop system model

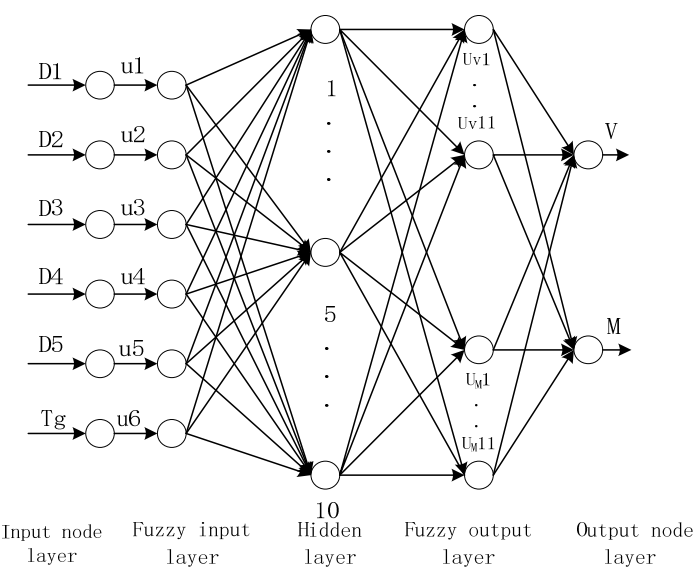

Fig.5 Fuzzy neural network 
Fuzzy BP neural network system. To reduce the complexity of the system, this paper designs a fuzzy neural network system with 6 input and 2 output which consists of five parts, as shown in Fig.5.

The first layer is the input node layer, and the input state space vector ' $\boldsymbol{x}$ ' $(7)$ is formed by the surrounding environment information that the sensors perceive. The nodes of this layer are connected directly with the components 'D1', 'D2', 'D3', 'D4', 'D5' and 'Tg' of the input state space vector ' $\boldsymbol{x}$ ' then pass it to the next layer.

The second layer is the fuzzy input layer, which fuzzifies the input vector, that each language variable is represented by a node. The input distance information 'D1', 'D2', 'D3', 'D4', and 'D5' are classified into two levels: 'I' (within the safety distance) and ' $O$ ' (outside the safety distance) by comparing the current distance with the safety distance 'SD'(11). The input angular amount ' $\mathrm{Tg}$ ' is blurred into five levels ('LB', 'LM', 'ZO', 'RM', 'RB'), and the universe of the domain (-180 ', $\left.180^{\circ}\right)$ is fuzzified and the vector sent to the hidden layer is $u=[\mu 1 \mu 2 \ldots \mu 6]$.

The third layer is hidden layer in which the final number of nodes is 10 hidden layers according to 0.618 segmentation selection method[11] of BP neural network and several experiments. In this paper, we choose the Sigmoid function " $S(x)=1 /\left(1+e^{-x}\right)$ " as the transfer function of hidden layer nodes, Then the output of the 'kth' hidden layer $\mathrm{y}_{\mathrm{k}}$ is expressed as “ $y_{k}=S\left(\sum_{i=1}^{p} w_{i k} \mu_{i}\right)$ ", Where $\mathrm{w}_{\mathrm{ik}}$ denotes the connection weights between the 'ith' fuzzy input node and the ' $k$ th' hidden layer node.

The fourth layer is fuzzy output layer. In order to facilitate the understanding of the output and operation, the output variable is transformed to the universe $[-1,1]$. The transformation formula is:

$$
g=\frac{2}{d-c}\left[x-\frac{c+d}{2}\right]
$$

Where [c, $\mathrm{d}$ ] is the domain of the actual output, and the variable ' $\mathrm{x}$ ' is transformed into the variable ' $g$ ' in the domain $[-1,+1]$. Each node of this layer corresponds to a linguistic variable of output. The membership of the output in different linguistic variables needs to be calculated by the membership function. The linguistic quantization subset is represented by the membership function ' $\mu_{\mathrm{v}}$ ' and ' $\mu_{\mathrm{m}}$ 'on the quantized space. In this paper, we use the Gaussian function as the membership function, which is expressed as:

$$
\mu_{i j}\left(x_{i}\right)=\exp \left(\frac{\left(x_{i}-\beta_{i j}\right)^{2}}{\delta_{i j}^{2}}\right)(i=1,2, \ldots n ; j=1,2, \ldots m)
$$

Where ' $\beta_{i j}$ 'and ' $\delta_{i j}$ 'represent the center and width of the Gaussian function, ' $\mathrm{n}$ ' is the number of input nodes, and ' $\mathrm{m}$ ' is the number of linguistic nodes of the input. In this paper, we divide the domain $[1,1]$ of the output variables ' $\mathrm{V}$ ' and ' $\mathrm{M}$ ' into 11 steps and output the signal format " $\mathrm{y}=\left[\mu_{\mathrm{v}}\left(\mathrm{c}_{1}\right) \mu_{\mathrm{v}}\left(\mathrm{c}_{2}\right) \ldots \mu_{\mathrm{v}}\left(\mathrm{c}_{11}\right) \mu_{\mathrm{m}}\left(\mathrm{c}_{1}\right) \mu_{\mathrm{m}}\left(\mathrm{c}_{2}\right) \ldots \mu_{\mathrm{m}}\left(\mathrm{c}_{11}\right)\right]$ ", where the values of " $\mathrm{c}_{1} 、 \mathrm{c}_{2} 、 \ldots \mathrm{c}_{11}$ " are " -1 , $-0.8, \ldots 1$ " respectively.

The fifth layer is the output node layer, which achieve the de-fuzzy function of the output. The common de-fuzzy method are area averaging method, area center method, maximum membership method, median method and weighted average method[12]. This paper uses the weighted average method, which is widely used in industrial control. The formula is:

$$
Z_{p}=\frac{\sum_{i=1}^{n} z_{i} \mu_{c}\left(z_{i}\right)}{\sum_{i=1}^{n} \mu_{c}\left(z_{i}\right)}
$$

The de-fuzzy formulas of output speed ' $\mathrm{V}$ ' and steering angle ' $\mathrm{M}$ ' are:

$$
\begin{aligned}
& V=\frac{(-1) * \mu_{v}(-1)+(-0.8) * \mu_{v}(-0.8) \ldots+\mu_{v}(1)}{\mu_{v}(-1)+\mu_{v}(-0.8) \ldots+\mu_{v}(1)} \\
& M=\frac{(-1) * \mu_{m}(-1)+(-0.8) * \mu_{m}(-0.8) \ldots+0.8 \mu_{m}(1)}{\mu_{m}(-1)+\mu_{m}(-0.8) \ldots+\mu_{m}(1)}
\end{aligned}
$$

The speed and steering angle of the hexapod robot are obtained by output space vector ' $s$ ' (10), in 
which ' $\mathrm{V}$ ' is divided into three grades: ' $\mathrm{Fa}$ ' $(30 \mathrm{~cm} / \mathrm{s})$, 'Lo' $(15 \mathrm{~cm} / \mathrm{s})$ and 'Ze' $(0 \mathrm{~cm} / \mathrm{s})$, and 'M' is divided into five levels as the hexapod robot walking output steering angle: 'TLL'(left turn $60^{\circ}$ ), 'TL'(left turn $30^{\circ}$ ), 'TF' (straight), 'TR' (right turn $30^{\circ}$ ), 'TRR' (right turn $60^{\circ}$ ). In Eq. $(15,16)$, Where ' $\mu_{\mathrm{v}}$ ' is the membership degree of the quantized subset of the output language ' $\mathrm{V}$ ', and ' $\mu_{\mathrm{m}}$ ' is the membership degree of the quantized subset of the output steering angle ' $M$ ' language.

\section{Simulation Experiment}

In order to validate the effectiveness and reliability of the hexapod robot autonomous navigation control system, it is simulated on MATLAB. A two-dimensional coordinate system is established to simulate sensors on the hexapod robot to detect obstacles and target point information in the unknown environment. Then the information is input to the navigation control system to calculate the traveling speed ' $\mathrm{V}$ ' and corner ' $\mathrm{M}$ ' which are used to control the robot to walk. The dots mark the position information of robot per second. The curve of small dots represents the robot's walking path, and the distance between adjacent dots represents the speed value, which with a longer distance means a higher speed. The starting point $A$ of robot is $(0 \mathrm{~m}, 0 \mathrm{~cm})$, the target point $B$ is $(1500 \mathrm{~cm}, 1500 \mathrm{~cm})$, and the range of traveling speed is $[0,30 \mathrm{~cm} / \mathrm{s}]$.

The open-loop and closed-loop navigation systems of the robot are simulated respectively which is shown in Fig. 6 and Fig.7. We can see that the robot can both safely bypass the obstacle to reach the target point in the open-loop system and closed-loop system, but the robot's walking path in the open-loop system is redundant relative to the closed-loop system. The simulation results show that the travel time of the robot in the closed-loop system is $94 \mathrm{~s}$, which is $8.74 \%$ less than the $103 \mathrm{~s}$ in the open-loop system.

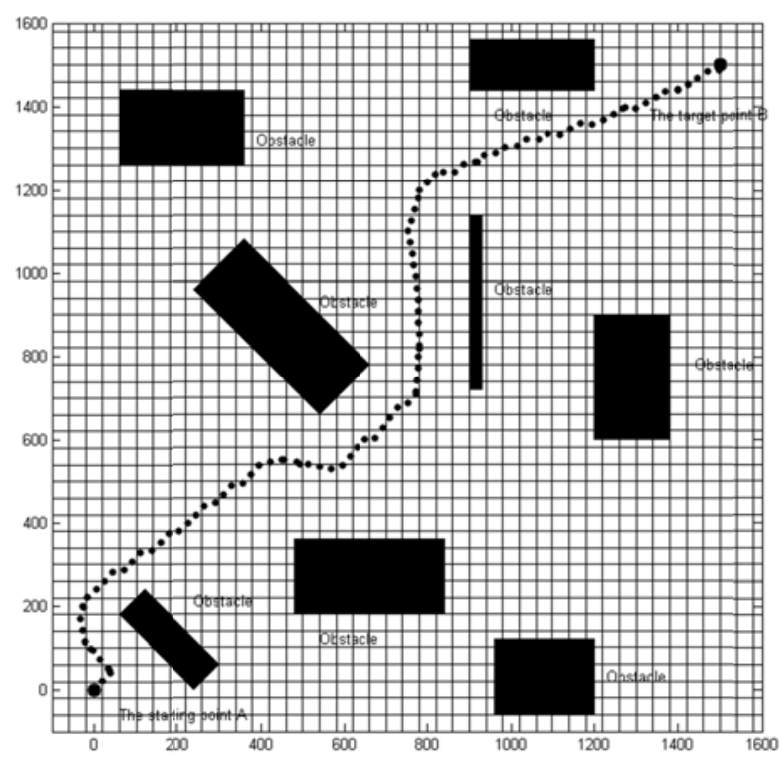

Fig.6 Simulation diagram of open-loop system

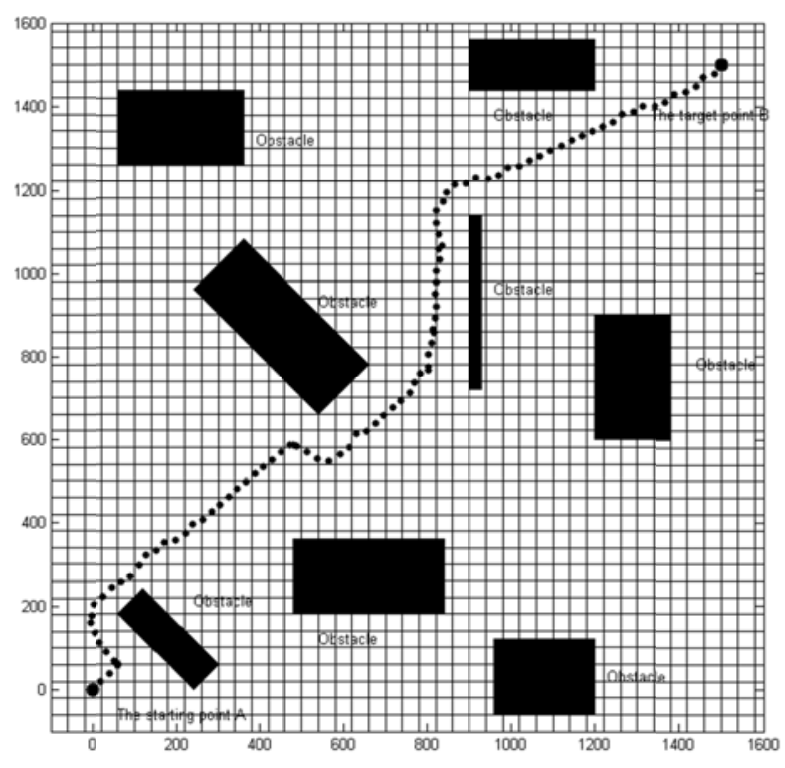

Fig.7 Simulation diagram of closed-loop system

\section{Conclusion}

In this paper, a closed-loop control autonomous navigation system based on fuzzy neural network is proposed to deal with the autonomous navigation issue of hexapod robot in unknown environment. Navigation algorithm used in the system combines the advantages of neural network and fuzzy control. The main innovations are as follows:

(1) The system uses a closed-loop control with feedback function that the output performance has been optimized compared to open-loop control system.

(2) Obstacle distance information of the system is obtained by ultrasonic sensor sector scanning which reduces the number of sensors and avoids the errors caused by beam angle interference and signal crosstalk. 
In this paper, the results of simulation experiment show that the closed-loop performance is better than that of open-loop. The closed - loop fuzzy neural network control system designed in this paper reduces the travel time of the robot and increases the average speed of the robot compared with the open-loop control system, and it can better achieve the autonomous navigation function of the hexpod robot in unknown environment. This paper also has some reference value for other types of autonomous navigation design of robot.

\section{Acknowledgements}

Authors wish to thanks the whole group members. This work is supported by the Jilin Province Science and Technology Development Plan Project "20140101224JC".

\section{References}

[1]Nichols E, Mcdaid L J, Siddique N H. Case study on a self-organizing spiking neural network for robot navigation.[J]. International Journal of Neural Systems, 2010, 20(6):501-8.

[2]Luo C, Yang S X. A Bioinspired Neural Network for Real-Time Concurrent Map Building and Complete Coverage Robot Navigation in Unknown Environments[J]. Neural Networks IEEE Transactions on, 2008, 19(7):1279-1298.

[3]Harisha S K, Ramkanth K P, Krishna M, et al. Fuzzy Logic Reasoning to Control Mobile Robot on Pre-defined Strip Path[J]. Proceedings of World Academy of Science Engineering \& Technolog, 2008:753.

[4]Masmoudi M S, Krichen N, Masmoudi M, et al. Fuzzy Logic Controllers Design For Omnidirectionnal Mobile Robot Navigation[J]. Applied Soft Computing, 2016, 49:901-919.

[5]Joshi M M, Zaveri M A. Optimally Learnt, Neural Network Based Autonomous Mobile Robot Navigation System[J]. International Journal on Control System \& Instrumentation, 2011.

[6]Borovic B, Liu A Q, Popa D, et al. Open-loop versus closed-loop control of MEMS devices: choices and issues[J]. Journal of Micromechanics \& Microengineering, 2005, 15(10):1917-1924.

[7]Shérine M. Antoun, Phillip J. McKerrow. Wall Following with a Single Ultrasonic Sensor.[C]// International Conference, Icira, Shanghai, China. 2010:130-141.

[8]Hua Liang, Feng Hao, Gu Ju-ping, et al. Wall following navigation strategy for mobile robot using single ultrasonic sensor[J]. Journal of Engineering Design, 2008, 15(3):206-212.

[9]Qingquan Li, Zhixiang Fang, Hanwu Li, et al. Integrating GPS, GYRO, vehicle speed sensor, and digital map to provide accurate and real-time position in an intelligent navigation system[J]. Proceedings of SPIE - The International Society for Optical Engineering, 2005, 6045:60451F-60451F-11.

[10]Pan Chang-hong, Chen Wei-dong, Xi Yu-geng. A neural network approach for safety path planning of mobile in unknown environments[J]. Acta Automatica Sinica, 2004, 30(6):816-823.

[11]Wen Jing-jun, Hua Xi-sheng. Improved Application of BP neural network to dam safety monitoring[J].Hydro power Automation and Dan Monitoring, 2006, 30(4):62-65.

[12]Cai Jian-xian, Ruan Xiao-gang, Gao Yuan-yuan. Stochastic fuzzy control strategy and application to robot control[J]. Electric Machines and Control, 2009, 13(5):754-761. 